\title{
Computer tools for spatial chromosome contacts analysis by ChIA-PET and Hi-C data
}

\author{
Dergilev A.I. ${ }^{*}$, , Luzin A.N. ${ }^{1}$, Kovalev S.S. ${ }^{2}$, Babenko R.O. ${ }^{1}$, Orlov Y.L. ${ }^{1,2}$ \\ ${ }^{1}$ Novosibirsk State University, Novosibirsk, Russia \\ ${ }^{2}$ Institute of Cytology and Genetics, SB RAS, Novosibirsk, Russia \\ *e-mail: arturd1993@yandex.ru
}

Key words: genome, transcription factor, medical informatics, ChIP-seq

Motivation and Aim: 3D contacts and interactions in interphase nuclei of eukaryotic cell play critical role for gene expression regulation. Series of post-genome technologies have been developed to study the binding of transcription factors for transcription regulation, such as chromatin immunoprecipitation arrays (ChIP-Seq) [1]. Correspondingly, set of software tool for processing of such data has been developed. Identification of genomewide distal chromatin interactions that lead the regulatory elements to their target genes may provide novel insights into the study of transcription regulation. Chromatin Interaction Analysis with Paired-End-Tag sequencing (ChIA-PET) method for such analysis requires development of specialized software [2]. The aim of the work was to review existing tools for 3D genome structure and spatial topological domains analysis. Methods and Algorithms: The data have been obtained via available data sources containing experimental information from ChIP-seq, Hi-C, ChIA-PET tests using different sequencing platforms. Gene annotation was obtained from UCSC Genome Browser. We reviewed existing software and created a database prototype of bioinformatics tools for 3D genome structure analysis.

Results: We tested program for analysis of ChIA-PET experimental data. The result of the program is a distribution of CTCF transcription factor binding sites on domains on the human chromosomes. The distributions of human genes relative CTCF binding sites and a randomly generated list of such sites as the program output were used to estimate statistical significance of the associations found.

Conclusion: With the rapidly increasing resolution of $\mathrm{Hi}-\mathrm{C}$ datasets, the size of the chromatin contact map will soon exceed the memory capacity of general computers. ChIA-PET and Hi-C technologies provide huge volume of data demanding development of new computer tools in different applications.

Acknowledgements: The work was supported by the RFBR and ICG SB RAS budget project (0324-0019-0040).

\section{References}

1. Li G. et al. Chromatin Interaction Analysis with Paired-End Tag (ChIA-PET) sequencing technology and application. BMC Genomics. 2014;15(Suppl. 12):S11.

2. Fullwood M.J. et al. An oestrogen-receptor-alpha-bound human chromatin interactome. Nature. 2009;462(7269):58-64. 